\title{
Ein bisher ungenannter Fink Westafrika's
}

\author{
Von
}

\section{Dr. Carl Bolle.}

\section{Crithagra Hartlaubii, C. Bolle n. sp.}

Supra olivaceo-viridis flammis nigricantibus obsolete notata; pileo laete cinereo nigro substriolato; superciliis latis in fronte confluentibus genis uropygio corporeque toto subtus pulchre flavis; stria utrinque mysticali cinerea; alarum tectricibus fusco-nigricantibus marginibus late flavescenti-viridibus fasciam alarem inconspicuam efficientibus; remigibus reetricibusque fusco-nigricantibus extus flavescenti, intus una cum rectrieum apiee dilute albido-marginatis; subalaribus flavis; pedibus cum maxilla obscure carneis; mandibula eornea, iride brunnea.

Foem. Omnino paulo pallidior, praesertim colore flavo magis in citrinum vergente; corporis lateribus olivaceo adumbratis quo colore pectoris citrinum a gulae leviter seiungitur; pedes carneo dilutiori.

Jun. av. Cum adulta congrua, coloribus omnibus autem multo pallidioribus sordidioribusque; superciliis, genis, corporeque inferiore albidoflavis; lateribus olivaceis cum fascia quadam inter pectus gulamque posita.

Long. circ. $4^{\prime \prime}$. - Rostr. $3^{1} / 4^{\prime \prime \prime}$. - Al. $2^{\prime \prime} 4^{\prime \prime \prime}$. - Caud. $1^{\prime \prime}$ $5^{\prime \prime \prime}$. - Tars. $5^{\prime \prime \prime}$ *).

Synon: Crithagra chrysopyga Hartl. Syst. Orn. W. Afr. Edit. I. p. 154; nec Swainson.

Grösse des Zeisigs etwa, aber mit stärkerem Kopfe und Halse. Schnabel, obwohl etwas länglich, doch mehr pyrrhulaartig gewölbt als beim Kanarienvogel. Zehen und Nägel mässig stark. Die Flügel erreichen etwa ein Drittel der Länge des am Ende nicht ausgerandeten Schwanzes und scheinen mehr einen Strich- als einen Standvogel anzudeuten. Stirn und Wölbung des Oberkopfes sehr flach, wie bei $\boldsymbol{C}$. butyracea, von welchem unser Vogel sonst in vielen Stücken ein wahres Miniaturbild abgiebt.

Diesmal ist es der Vogelhandel und die oft getadelte Liebhaberei; denen die Systematik eine kleine Bereicherung zu danken hat. Es scheint kaum glaublich, dass ein Vogel, der seit Jahren schon in einiger Menge lebend nach Europa gebracht wird, sich als neue Species herausstellen sollte und doch ist dem so und er dürfte vielleicht nicht der einzige

*) Die Maasse nuch Hartlaub, da so zarte Thierchen sich lebend nicht wohl messon lassen. 
sein, den die übers Meer gebrachten Transport-Käfige von Senegal und Rio-grande noch unbeschrieben hinter ihren Gittern bergen. Seine bisherige Vernachlässigung beruhte auf einer Verwechslung mit der sehr nahe verwandten Crithagra chrysopyga Swainsons. Genauere Vergleichung mit dieses Autors Abbildung und Beschreibung, Birds of West. Africa I. p. 206 t. 17, lehrt jedoch unfehlbar, dass der daselbst aufgestellte Vogel mit dem, dessen Diagnose wir so eben nach lebendigen Exemplaren gaben, nicht identisch sein kann. Der hell-aschgraue Oberkopf und Nacken, welcher beiden Geschlechtern von C. Hartlaubii eigen ist, genügt schon zur specifischen Sonderung letzterer, indem die echte Swainsonsche Art am ganzen Oberkörper, von der Stirn bis zum Steisse exclusive, ein gleichmässig olivenfarbiges Kleid trägt. Herr Dr. Hartlaub, dem dieser Unterschied nicht entgangen war, hatte bisher Swainsons Abbildung für die eines jüngeren Exemplars gehalten, welches die graue Kopfzeichnung noch nicht tragen möchte. Dass der Verfasser der „Birds of Western Africa“ dieselbe übersehen haben sollte, hält er für geradezu undenkbar. Es bleibt daher nur übrig eine zweite westafrikanische Art anzunehmen, um so mehr, da ich mich positiv davon überzeugt habe, dass schon der jüngere Vogel das Grau der Kopfzeichnung trägt.

Die Sache verhält sich folgendermaassen. Nachdem ich 1855 und 56 eine gewisse Anzahl lebender Exemplare bei den Vogelhändlern Berlins sowohl als in Teneriffa, ohne indess den Namen der Species zu kennen, beobachtet hatte, gelangte ich im Frühling 1857 in den Besitz eines Pärchens im Jugendkleide, welches ich im Hochsommer durch Vermauserung in die vollkommne Tracht übergehen sah und noch in diesem Augenblick lebendig besitze. So konnte ich es über allen Zweifel erheben, dass schon das erste Gefieder vollkommen demselben Grundtypus wie das der Erwachsenen nachgebildet sei. Nur sind sämmtliche Farben bei Weitem blasser, unreiner und verwaschener; namentlich zeigt sich das Gelb überall viel weniger markirt und wird durch die graugrüne Färbung der Seiten, so wie durch eine Art olivenfarbenen Bandes zwischen Brust und Kehle, nebst einigen Flecken daselbst, beeinträchtigt. Das Graue des Kopfes aber tritt, wenn auch lichter, doch ganz entschieden hervor. In der Nackengegend verschmelzen bei Alt und Jung die Farbentöne von Kopf und Rücken so allmälig, dass sie ohne scharfe Scheidelinie in einander übergehen.

Mit einigen meiner Berliner ornithologischen Freunden hatte ich diesen hübschen kleinen Exoten zuerst, einer irrigen vagen Bestimmung zufolge, Fringilla flaviventris genannt. Im Herbste des verflossenen 
Jahres nun sah ich Exemplare desselben in der überaus reichen Sammlung des Bremer Museums, nachdem mich kurz vorher schon Herr Dr. Hartlaub auf mein Anfragen brieflich davon in Kenntniss gesetzt hatte, es sei $C$. chrysopyga.

Da dieser Vogel seitdem Gegenstand mehrfacher Correspondenz zwischen mir und dem eben genannten ausgezeichneten Forscher gewesen ist, dessen Erläuterungen ich es verdanke, zu der Ansicht gekommen zu sein, dass es entschieden zwei westliche Crithagras giebt, (zu welcher von beiden Heuglin's Dryospiza aurifrons aus 0st-Sennaar gehöre, bleibt vor der Hand dahingestellt) so möge es mir vergönnt sein, die noch unbenannte Art, mit einem Namen zu belegen, der mit deutscher Forschung auf dem Gebiet exotischer, namentlich aber westafrikanischer Ornithologie fast gleichbedeutend geworden ist.

Da Crithagra Hartlaubii, im Sinne Bonaparte's wohl ein Serinus, ihren Weg in nicht geringer Anzahl lebend nach Europa findet, während wir daselbst den Swainsonschen Vogel noch nie sahen, so dürfte der ersteren Vorkommen längs der Sumpfküsten und an den grossen Strömen des tropischen West-Afrika's leicht das häufigere sein. In dé Gefangenschaft scheint sie bisher unter dem jedenfalls mehr als eine Species umfassenden Begriff des "Serin de Mozambique" rangirt zu haben. Wenn einmal die Frage, welchem Vogel eigentlich dieser letztere, schon alte aber dunkle Name (wahrscheinlich giebt es an Afrikas 0stund Westküste wenigstens drei verschiedene Arten dieser Gruppe) zukommt, endgültig gelöst sein wird, dann erst wird auch auf die Abgrenzung unserer Species ein helleres Licht fallen. Bis jetzt fehlt es uns noch vollkommen an Aufschluss, sowohl über deren bestimmtere geographische Verbreitung, als über deren Lebensweise im Vaterlande; will man nicht etwa auf sie die in der Corografia Caboverdiana gegebene kurze Notiz Chelmicki's beziehen: die Wälle der Festung Bissae im portugiesischen Guinea seien von Tausenden von Crithagra chrysopyga bedeckt.

In Ermangelung jedweder Nachricht über die Sitten dieser Art im Zustande der Freiheit, wollen wir wenigstens ein Paar Worte über dieselbe als Stubenvogel hier Platz finden lassen. Für die Voliere ist Crithagra Harllaubii *) eine äusserst wünschenswerthe Acquisition. Seine

*) Einen deutschen Namen bat der Vogel bisher noch nicht erhalten; man müsste denn das barbarische Wort „Mozambek“, mit dem ich ihn mehrmals von denen, die ihn herübergebracht, belegen hörte, für einen solchen nehmen. Er ist

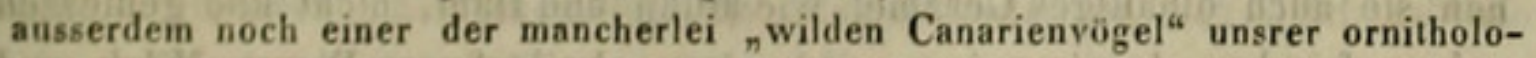
gisch nicht grade sehr gebildeten Vogelhändler. 
zierliche Gestalt und seine wahrhaft anmuthige Färbung werden dnrch ein ruhiges und gewandtes, obwohl nicht grade auffallend zutrauliches Wesen noch mehr hervorgehoben und gern vergisst man darüber das Fehlen eines eigentlichen Gesanges. Die Stimme des Vogels besteht in einem häufig ausgestossnen leisen Zirpen; selten hört man dazwischen einen kanarienvogelartigen gedehnt flötenden Ton. Der Lockruf ist aus drei lauten und wohlklingenden absteigenden Noten die schnell und unmittelbar aufeinander folgen, zusammengesetzt. Mit diesem locken die Pärchen, wenn man sie trennt, einander unaufhörlich. Paarweis gehalten, thun die stets sauberen und schmucken Thierchen sich gern eng zusammen, ohne sich jedoch so innig wie die Estrelden, aneinander zu schmiegen, und legen dabei untereinander die grösste Zärtlichkeit an den Tag. Es sind starke Degorgeurs; fortwährend schnäbeln und füttern sie sich aus dem Kropfe und zwar in den zierlichsten Stellungen, die in den Juli fallende Mauserzeit allein ausgenommen. Trotzdem zeigen sie gar keine Neiguug sich in der Gefangenschaft fortzupflanzen oder ein Nest zu bauen, wie hoch auch die Sommerwärme steigen möge und wie weit auch der Raum sei, den man ihnen anweist. Wahrscheinlich fällt die Zeit dazu in Senegambien in eine von unserem Sommer ganz verschiedene Jahreszeit. Zu Santa Cruz de Tenerife war ich Zeuge eines vergeblichen Versuchs, den man machte, ein Weibchen von Hartlaubii mit einem Canarienvogel zu paaren. Dagegen beobachtete ich eben daselbst in meines Wirthes D. Diego Guerin's wohlbesetzter Voliere ein intimes Freundschafts-Bündniss zwischen unserem Vogel und einem schon sieben Jahr den Käfig bewohnenden überaus niedlichen Tomeguin von Cuba (Euethia canora Cab. Pyrrhula collaris Vig.), der, obwohl kaum grösser als ein Zaunkönig, doch die viel ansehnlichere Crithagra, zu der er sich wegen beiderseitiger Vereinsamung gesellt, aufs Liebevollste zu füttern pflegte. - Zur Nachtruhe ziehen diese Vögel die höchsten ihnen erreichbaren Stellen vor, schlafen auch oft, wie die Zeisige, seitwärts am Gitter ihres Bauers angeklammert; sonst gern neben einander auf einer Stange sitzend. Auf die Erde kommen sie meist nur herab um zu fressen oder das Wasser aufusuchen, in welchem letzteren sie sich beim Baden sehr durchnässen. Eine besondere Empfindlichkeit gegen kühlere Luft habe ich nicht an ihuen wahrgenommen, obwohl sie im Winter natürlich eines gut geheizten Zimmers bedürfen. Ihre Nahrung besteht aus mehlreichen Sämereien, unter denen Hirse und Canariensamen ihnen besonders zuzusagen scheinen; doch verschmähen sie auch ölhaltiges Gesäme, wie Mohn und Hanf, nicht und geniessen neben frischen Ameiseneiern und einem beiläufigen Bissen Milch und 
Semmel, gern zartes, junges Grün: eine Neigung, in welcher sie, wie in ihren Sitten überhaupt, mit den meisten andern "Serins" zusammentreffen.

Berlin, am 5. Juli 1858.

\section{Bemerkungen, Beobachtangen und Anfragen.}

\section{Von}

F. Boie, in Kiel.

\section{Sippe. Rubigula Blyth,}

Turdus dispar Horsfield finde ich Mus. Heineanum p. 110 als Sippe Sphagias 1851 gesondert, eine Benennung, welche der älteren Rubigula Blyth weichen dürfte. Als zweite Art kommt Brachypus rubineus Jerdon in Betracht, bei beiden als Sonderungsgrund die gelbe Iris, die freilich nicht als Sonderungsmerkmal zu benutzen.

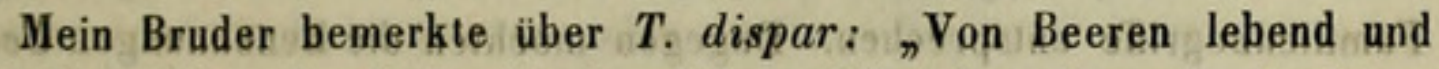
im dichten Gebüsch. Ruft pipi piet glick, und hat darneben einen Lockton, welcher dem von Fringilla carduelis ähnlich ist. Rachen wachsgelb.

2. Sippe. Platypus Brehm und Lusciala Blasius.

Beide stellen sich mir als Sonderungen nach dem Vorbilde älterer Genera dar, die im natürlichen Systeme eben so wenig als Sippe wie als Familie validiren, in ersterer Bedeutung zu viel, in letzterer zu wenig umfassen.

\section{Sippe. Lalage.}

Ich nannte 1826 als deren Typus Turdus orientalis, meinte aber den Turdus mindanensis, den ich irrthümlich mit ersterem für identisch hielt, ich entsinne mich nicht durch welche Autorität misleitet. Der Irrthum hatte zur Folge, dass später Gray meine Gruppe den Campephagiden unterordnete, während Sundevall Utdrag us Kongl. Vetenskaps-Academiens Handlingar p. 89 sie in dem von mir gewünschten Sinne aufgefasst, auf den der Name hindeutete.

$$
\text { 4. }
$$

Soll derjenige, welcher zuerst eine Art oder eine Reihe von Arten generisch absonderte, oder, nach dẹr nöthig gewordenen Verwerfung einer älteren Benennung, der, welcher eine bessere substituirte als Autor bezeichnet werden?

Das letztgedachte Verfahren heisst der Form einen Vorzug vor der Idee einräumen, hat aber freilich auch Gründe für sich, Ich möchte einen Mittelweg einzuschlagen und z. B. 


\section{$2 \mathrm{BHL}$ Biodiversity Heritage Library}

1858. "Ein bisher ungenannter Fink Westafrika's." Journal fu

r Ornithologie 6, 355-359. https://doi.org/10.1007/bf02010059.

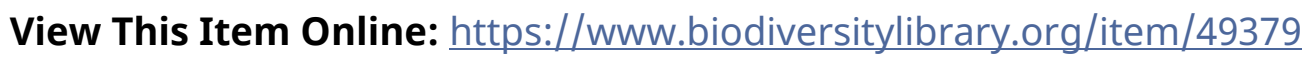

DOI: https://doi.org/10.1007/bf02010059

Permalink: https://www.biodiversitylibrary.org/partpdf/141368

\section{Holding Institution}

Natural History Museum Library, London

\section{Sponsored by}

Natural History Museum Library, London

\section{Copyright \& Reuse}

Copyright Status: Public domain. The BHL considers that this work is no longer under copyright protection.

This document was created from content at the Biodiversity Heritage Library, the world's largest open access digital library for biodiversity literature and archives. Visit BHL at https://www.biodiversitylibrary.org. 